\title{
Shear thickening effects of drag-reducing nanofluids for low permeability reservoir
}

\author{
Chunyuan $\mathrm{Gu}^{1 \oplus *}$, Rundong Qiu${ }^{1}$, Songlin $\operatorname{Liu}^{2 *}$, Zhenjiang You $^{3}$, Rongsheng Qin ${ }^{1}$ \\ ${ }^{1}$ Shanghai Institute of Applied Mathematics and Mechanics, Shanghai Key Laboratory of Mechanics in Energy Engineering, School of \\ Mechanics and Engineering Science, Shanghai University, Shanghai 200444, P. R. China \\ ${ }^{2}$ Oil production Plant 1 of Jiangsu Oilfield Branch of Sinopec Group, Yangzhou 225265, P. R. China \\ ${ }^{3}$ School of Chemical Engineering, The University of Queensland, Brisbane QLD 4072, Australia
}

\section{Keywords:}

Drag-reduction nanofluid

non-Newtonian fluid

shear thickening mechanism

constant index constitutive equation

Cited as:

Gu, C., Qiu, R., Liu, S., You, Z., Qin, R. Shear thickening effects of drag-reducing nanofluids for low permeability reservoir. Advances in Geo-Energy Research, 2020, 4(3): 317-325, doi:

10.46690/ager.2020.03.09.

\begin{abstract}
:
Drag-reducing nanofluids are complex non-Newtonian fluids. Their constitutive characteristics are the basis of flow mechanism analysis in porous media. However, the rheological effects of drag-reducing nanofluids have not been thoroughly studied. In the present work, rheological properties of several nanofluids were measured, and the shear thickening mechanism was investigated experimentally. The results show that all the nanofluids examined have complex characteristics and critical shear rates. The viscosity exhibits a slow linear increase with the shear rate below the critical shear rate, while the shear thickening power-law fluid behaviour appears above the critical shear rate. The critical shear rate increases with the increase of particle concentration, which indicates the injection rate needs to be controlled to avoid significant increase of nanofluids viscosity. The rheological curve of increasing shear rate nearly coincides with that of decreasing shear rate, which indicates that the shear thickening of nanofluids studied in this work is transient and reversible. A constant index constitutive equation with an exponent of 0.5 is obtained from test results by the fixed index method, and its coefficient $k(c)$ is a linear function of the concentration, which can replace a set of conventional constitutive equations with different concentrations. The constant index constitutive equation also clarifies the coefficient dimension. Similar results have been obtained by analysing several other nanofluids using the fixed index method, which validates the new effective method for constructing the constitutive equations of non-Newtonian nanofluids.
\end{abstract}

\section{Introduction}

Nanotechnologies have many advantages of solving the bottlenecks of chemical flooding and are expected to become a strategic technology for the next generation of enhanced oil recovery (Yuan and Wang, 2018; Moghadasi et al., 2019; Alnarabiji and Husein, 2020). Nanofluid drag reduction technology is a new method to solve the problem of "high injection pressure", which seriously affects the production and recovery from medium or low permeability reservoirs. It is one of the important directions to improve oil recovery nowadays.

A lot of researches have been carried out and great progress has been made in drag reduction mechanism, preparation and field application of drag reduction nanofluids (DRNFs) (Ju et al., 2002; Lu et al., 2003; Chen et al., 2016). Gu et al. (2007) proposed the drag reduction mechanism that the core wettability is reversed by competitive adsorption between nanoparticles and water film on rock surface and the water flow slip effect takes place. The superhydrophobic particle adsorption layer was constructed in adsorption experiments. Assef et al. (2014) have concluded that nanofluids (NFs) can inhibit the migration of clay particles and improve the low salinity and alkaline waterflooding outcomes. The preparation of nano-powder with different particle sizes and water-based NFs has also been greatly developed (Cheng et al., 2006; Cao et al., 2010; Yue, 2014) and drag reduction effect is remarkable in field applications (Hu et al., 2012; Wang et al., 2012; Yu et al., 2012). Some results also show that DRNFs can reduce oil-water interfacial tension, improve oil washing efficiency

Yandy
Scientific
Press

* Corresponding author.

E-mail address: wein1989@163.com (C. Gu); 1124148467@qq.com (R. Qiu); 1slin.jsyt@sinopec.com (S. Liu);

zhenjiang.you@gmail.com (Z. You); 1611225283@qq.com (R. Qin).

2207-9963 (c) The Author(s) 2020.

Received June 5, 2020; revised July 9, 2020; accepted July 9, 2020; available online July 17, 2020. 
(Zhao et al., 2018; Alnarabiji and Husein, 2020), and increase oil recovery by $5 \% \sim 15 \%$ compared with waterflooding. However, there are still many unsolved problems in rheology and transport mechanism of NFs.

Rheology is a scientific discipline of great utility in nanotechnology and provides many convenient tools to explore the influence of various physical and chemical factors on the properties of NFs (Mohamadian et al., 2018; Jóźwiak and Boncel, 2020). The rheology is very important for the flow characteristics and subsequent effects of DRNFs during injection into reservoir, which has not been paid much attention. The effect of flow rate on viscosity is usually ignored and critical parameter values are not yet available.

NFs are non-Newtonian fluid and the rheology is complex (Mohamadian et al., 2018; Navarrete et al., 2020). The rheology behaviour of NFs depends on various factors such as nanoparticle size (He et al., 2007; Jóźwiak and Boncel, 2020), nanoparticle concentration (Wang et al., 1999; Das et al., 2003), nanoparticle shape (Chen and Ding, 2009; Heine et al., 2010; Ettefaghi et al., 2013), nanoparticle structure (Baghbanzadeh et al., 2014), surfactant (Yang et al., 2006; Lu et al., 2007; Wang et al., 2013), shear rate (Alphonse et al., 2009; Aladag et al., 2012; Moghaddam et al., 2013) and even magnetic field (Hong et al., 2007; Susan-Resiga et al., 2012; Moattar et al., 2013). Muhammad et al. (2019) found at lower concentrations (approximately less than $23 \mathrm{vol} \%$ ), the relative viscosities of dispersive NFs show Newtonian behavior, and shear thinning occurred only for non-dispersive NFs. But shear thickening only occurs in highly concentrated NFs of more than $23 \mathrm{vol} \%$. NFs containing spherical nanoparticles can exhibit both types of behaviour: Newtonian at low shear rate values and non-Newtonian at high shear rate values (Sharma et al., 2016).

The non-Newtonian fluids are mainly divided into four categories: inelastic fluid (viscosity is not constant), fluid with yield stress, fluid with various elastic properties and thixotropic fluid. There are still many types in each category, corresponding to different constitutive equations (Chan and Mong, 1983). The respective mechanisms are also complex and not well understood.

The DRNF is a nanodispersion containing $\mathrm{SiO}_{2}$ nanoparticles dispersed in a specific liquid (Pang et al., 2016). Our results show that the DRNF is a very complex non-Newtonian fluid with overall shear thickening and local power law fluid characteristics. In the understanding of shear thickening mechanism, there are many viewpoints. The theory of order and disorder is one of the main theories, which states that in the early stage of action, the external force is small, the structure of the system is relatively stable, and the dispersed particles are arranged in order, so it leads to shear thinning. While the order is broken with the increase of external force, the disorder leads to shear thickening (Catherall et al., 2000; Sha, 2013). The theory of particle clusters is another major theory (Bossis and Brady, 1989; Petel et al., 2013). It is believed that the structure of the system has changed under the action of external force. When the external force is small, the van der Waals force or hydrogen bond between the particles rapidly recovers the damaged space structure, and the viscosity of the system does not change significantly. When the external force exceeds a certain critical value, the friction between the particles increases, the particles deviate from the equilibrium position and cannot return in time, so that they gather to form "particle clusters". In the flow process, when the relaxation time is longer than the convection time, the "particle clusters" will be formed and grow, resulting in higher viscosity. Maranzano and Wagner (2001) found that the microstructure of the system does accompany the change of hydrodynamic force to form a "particle cluster" during the shear thickening transition through small-angle neutron scattering experiments. Muhammad et al. (2019) also thought the origin of shear thickening is not the initial agglomeration but the structural formation of nanoparticles (NPs) under shear at high NP concentrations. Wang et al. (2013) reviewed literature available on rheology of NFs and found Brownian motion and nanoparticle aggregation to be the major mechanisms for rheological properties of NFs.

The constitutive equations of NFs are also the basis for the study of fluid mechanics and transport mechanism. The constitutive equation of the power-law flow of non-Newtonian fluid is $\mu=k \gamma^{n-1}$ or $\tau=k \gamma^{n}$. The exponent $n$ is a dimensionless constant, and the dimension of the coefficient $k$ depends on $n$. The parameters of $k$ and $n$ are related to the concentration of nanoparticles (Pang et al., 2016). But this equation does not show the concentration factor, so a set of equations are needed to represent different concentrations. Another problem is parameter dimension uncertainty. The exponent $n$ of different concentrations is different, so it causes uncertainty of the parameter dimension, resulting in the confusion of physical meaning. Obviously, this is very unfavorable for both problem analysis and equation solving.

Based on the DRNF HNFIII and two other NFs with hydrophilic $\mathrm{SiO}_{2}$, the rheological properties of NFs were studied to obtain the non-Newtonian types and critical parameters. The shear thickening effects were further discussed using increasing and decreasing shear rate experiments. A constant index constitutive equation (CICE) was established to replace a set of conventional constitutive equation (CCEs) of NFs with different concentrations using the fixed index method, clarify the dimension of the parameters and provide a new method for constitutive equation of non-Newtonian fluid. The rheology and shear thickening mechanism of NFs are studied, and the critical flow velocity is analyzed, which lays the foundation for elucidating the transport mechanism of drag-reducing NFs.

\section{Preparation and test methods of NFs}

The rheological properties of various NFs with shear rate were tested by Haake rotary rheometer RS-6000 (Thermo Fisher Scientific, USA). Outer diameter of rotor is $41 \mathrm{~mm}$. Inner diameter of stator is $43 \mathrm{~mm}$. The test temperature is set at $20{ }^{\circ} \mathrm{C}$.

Nanomaterials for experiment are $\mathrm{SiO}_{2}$ nano-powders prepared by Shanghai University. The size of $\mathrm{SiO}_{2}$ particles is about $30-40 \mathrm{~nm}$. HNPIII is a modified strong hydrophobic nano-powder. Three typical NFs were prepared: (1) A DRNF HNFIII used in the field (No. 1). The dispersion solution is a combination of various surfactant aqueous solutions. HNPIII 


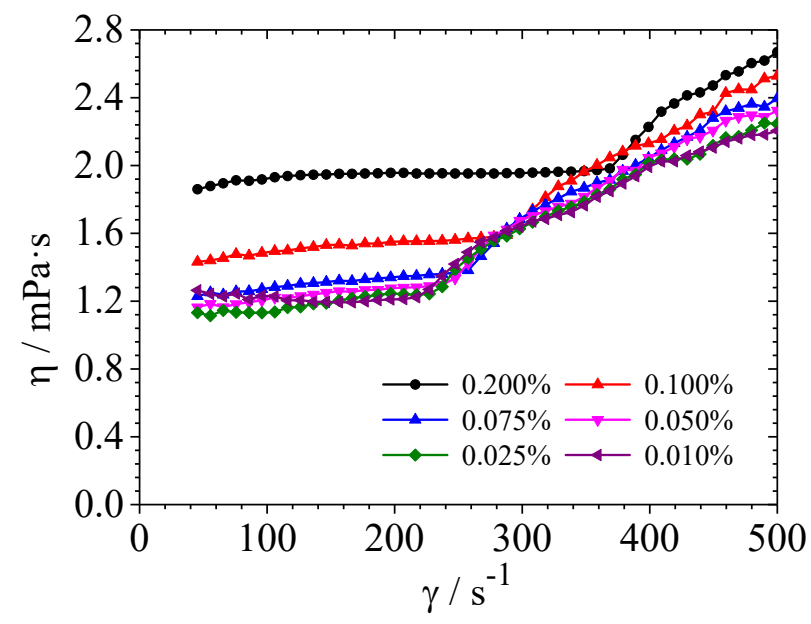

Fig. 1. The AV of NFs (No. 1) with shear rate at various concentrations $\left(0 \sim 500 \mathrm{~s}^{-1}\right)$.

particles were dispersed into dispersants and prepared into $11 \mathrm{NFs}$ of $0.2 \mathrm{wt} \% \sim 0.0025 \mathrm{wt} \%$. (2) A variety of $\mathrm{NFs}$ of $0.05 \mathrm{wt} \% \sim 0.15 \mathrm{wt} \%$ prepared from hydrophilic nanometer $\mathrm{SiO}_{2}$ dispersed to $1.0 \%$ surfactant SDS solution (No. 2). (3) A variety of $\mathrm{NFs}$ of $0.05 \mathrm{wt} \% \sim 0.15 \mathrm{wt} \%$ prepared from hydrophilic nanometer $\mathrm{SiO}_{2}$ dispersed to $1.0 \%$ surfactant STA solution (No. 3).

The rheological test method is as follows: first, take appropriate amount of NFs, and load it into the measuring tube. Then, set the test parameters, such as temperature and shear rate range. Finally, start the test. The shear rate was set from 0 to $3500 \mathrm{~s}^{-1}$ and the interval was $76 \mathrm{~s}^{-1}$. During unidirectional shear rheological test, the shear rate first ranged from 0 to 550 or $300 \mathrm{~s}^{-1}$, then dropped to 0 , and the interval was $6 \mathrm{~s}^{-1}$.

\section{Results and discussions}

\subsection{Characteristics of shear rheology of drag-reducing NFs}

Fig. 1 shows the rheological curves of NFs (No. 1) at different concentrations. The viscosity of NFs at different concentrations increases with shear rate, indicating that all the tested NFs have shear thickening characteristics, and this viscosity is called apparent viscosity (AV). There is an inflection point on the curve that corresponds to the critical shear rate (CSR) $\gamma_{0}$. When the shear rate is less than $\gamma_{0}$, the $\mathrm{AV}$ shows an insignificant linear increase trend with the shear rate, but the slope is small and the viscosity change is limited. So it can be regarded as Newtonian-like fluid. When the shear rate exceeds $\gamma_{0}$, the viscosity increases rapidly with the shear rate, showing obvious shear thickening phenomenon, which is the feature of power-law fluid. Therefore, NFs have the combined features of Newtonian and power-law fluids. The $\gamma_{0}$ values of NFs are $378 \mathrm{~s}^{-1}, 298 \mathrm{~s}^{-1}$ and $227 \mathrm{~s}^{-1}$ at the particle concentrations of $0.2 \mathrm{wt} \%, 0.1 \mathrm{wt} \%$ and $0.01 \mathrm{wt} \%$, respectively. These indicate that the critical shear rate is different for different concentrations of NFs. The higher the concentration, the greater the critical shear rate and the AV. The concentration is the dominant factor affecting the $\mathrm{AV}$ of NFs in the shear rate below $\gamma_{0}$, but the shear rate becomes the most important affecting factor after the shear rate exceeding $\gamma_{0}$.

In a word, the DRNF is a complex non-Newtonian fluid with the combined characteristics of Newtonian-like fluids and power-law fluids, which contribute to the injection and oil displacement. In injection process of DRNF, the smaller the viscosity, the easier the injection. It is advisable to inject by low flux. In the process of injecting water to replace NFs or NFs flooding, it is advisable to increase the flow rate properly. Thus, the shear thickening will help to reduce wateroil mobility ratio and improve the recovery. However, it is also necessary to prevent the blockage of nanoparticles in the pore throats or thin channels caused by high flow rate.

\subsection{Discussion on shear thickening mechanism of NFS}

Whether the shear thickening mechanism of NFs conforms to the disorder theory or the "particle cluster" theory (Bossis and Brady, 1989; Petel et al., 2013) is unclear. The reversibility of viscosity change was studied, and the shear thickening mechanism was analyzed by a double-direction shear test with increasing and decreasing of shear velocity. If the deceleration leads to a significant difference in viscosity, it indicates that the NFs may have a structural change, such as particle agglomeration. Otherwise, the structure does not change.

Figs. 2(a)-(d) present the bidirectional shear rheology of No. $1 \sim$ No. 3 NFs: A DRNF HNFIII of $0.15 \%$ (No. 1a), a hydrophilic $\mathrm{SiO}_{2}$ nanofluid of $0.15 \%$ (No. 2b), and two hydrophilic $\mathrm{SiO}_{2}$ NFs with two concentrations of $0.15 \%$ (No. $3 \mathrm{c}$ ) and $0.1 \%$ (No. 3d), respectively. The shear rate increases from 0 to $300 \mathrm{~s}^{-1}$ or $550 \mathrm{~s}^{-1}$, then decreases to 0 . The red curves represent the shear rate increase, and the green curves represent the shear rate decrease in Fig. 2.

All the NFs tested have similar shear thickening properties in Fig. 2. The forward and reverse shear rheological curves basically coincide, and the values of critical shear rate are the same, i.e., their rheology exhibits shear reversibility. The AV decreases with shear rate decreasing, which indicates that the nanofluid structure not only does not change irreversibly, but also can quickly recover to its initial state.

The same type of NFs at various concentrations have shear thickening and reversibility, indicating that the concentration has no effect on the essential properties of the NFs.

The reversibility of the fluid indicates that the nanofluid is only a transient physical recombination during shearing without irreversible structural changes. From the mechanical point of view, the force between liquid and nanoparticles can still be balanced at low shear rate, and the particles flow synchronously with liquid without relative motion, and the viscosity increases slowly or remains unchanged. When the shear rate exceeds the critical value, the equilibrium between liquid and nanoparticles is broken and the velocity of the base liquid is no longer consistent with that of particles. The particles are separated from the base liquid and form aggregates, accompanied by the turnover and collision of the 


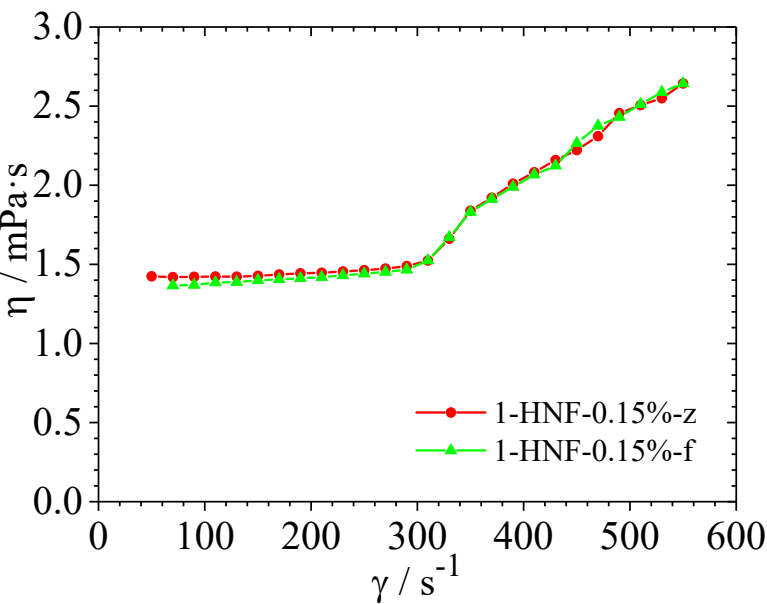

(a)

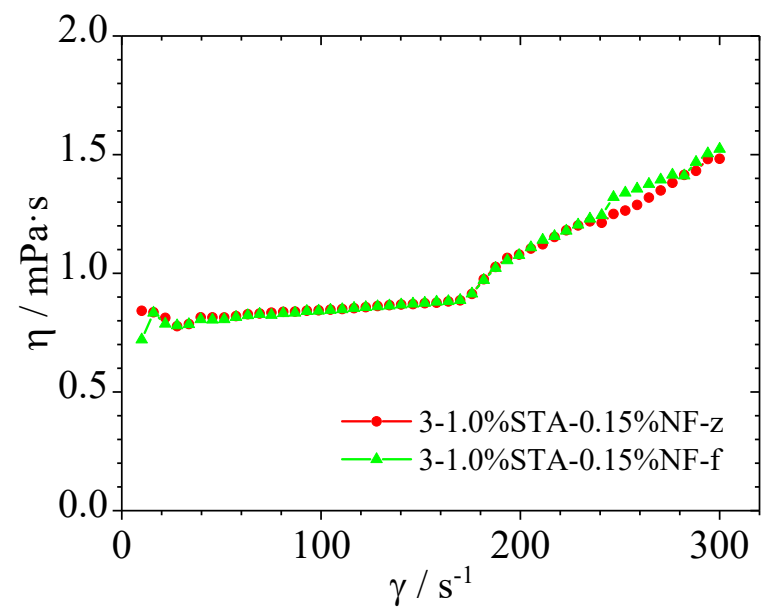

(c)

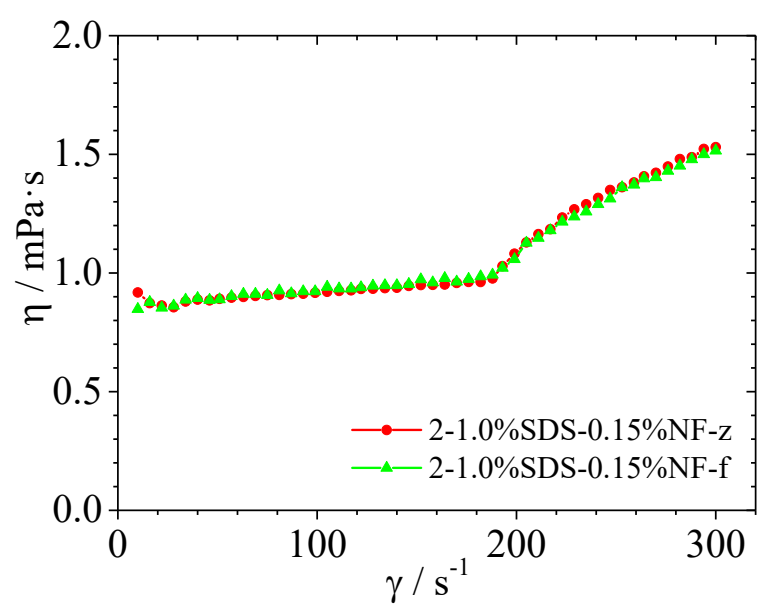

(b)

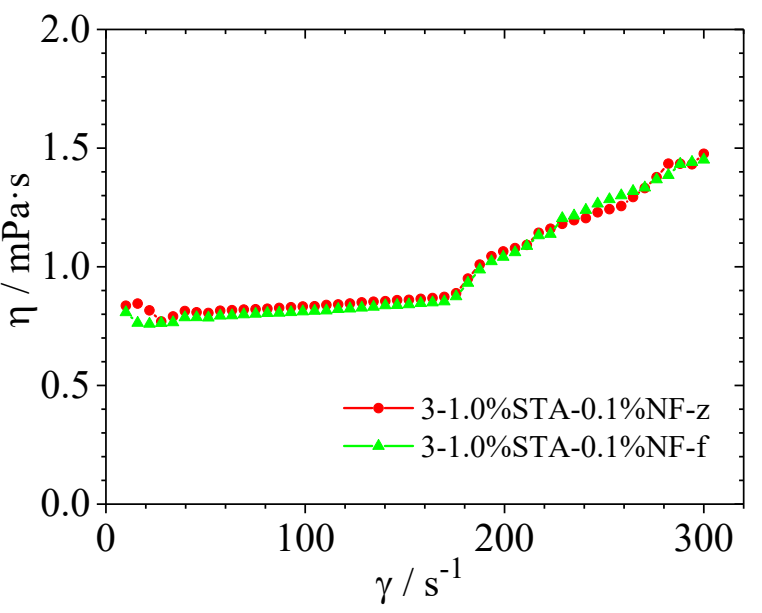

(d)

Fig. 2. The bidirectional shear rheology of NFs: (a) DRNF HNFIII of $0.15 \%$ (No. 1); (b) $\mathrm{SiO}_{2}$ nanofluid of $0.15 \%$ (No. 2); (c) nanofluid of $1.0 \% \mathrm{STA}+0.15 \% \mathrm{SiO}_{2}$ (No. 3); (d) nanofluid of $1.0 \% \mathrm{STA}+0.1 \% \mathrm{SiO}_{2}$ (No. 3).

particles, which leads to a sharp increase in viscosity and local inhomogeneity of the fluid. When the shear rate is reduced, the additional shear external force decreases and transforms to equilibrium. The velocity difference between the particle and the base liquid decreases or eliminates, the motion tends to be consistent, and so the viscosity decreases.

The aggregates are the so-called "cluster of particles" observed by Maranzano et al. (2001) in scattering experiments. However, it is clear that there is no stable binding force in the "cluster of particles" because once the shear force decreases or disappears, the particles will disperse and return to their original state, which is evidenced by the reversibility of rheology.

In summary, there are two stages in the shear process of $\mathrm{NFs}$, one is the synchronous and orderly flow of the base liquid and the particles, which is Newtonian-like fluid flow. The other is that the particles are separated from the base liquid and aggregate into loose "particle clusters". The phenomenon from Newtonian-like fluid to fast shear thickening is similar to the transition of fluid flow from laminar to turbulent, which is just a change in fluid flow, not an internal structure.

\subsection{The non-Newtonian types of NFs HNFIII}

Fig. 3 and Fig. 4 are the shear rate-viscosity curves of NFs before and after the critical point, respectively.

The curves show the AV has a very slow linear increasing relationship with the shear rate before reaching the critical point in Fig. 3. The increasing slope has the order of $10^{-4}$ only, and the maximum increase is no more than $15 \%$. Therefore, it can be considered as a Newtonian-like fluid before reaching the critical shear rate, and the AV can be regarded as a constant, but its value is related to the concentration.

The AV increases rapidly with the shear rate after the critical point, and all the curves follow the exponential relationship in Fig. 4, which satisfies the equation $\eta=k \cdot \gamma^{n-1}$ or $\tau=\eta(\gamma) \cdot \gamma=\gamma^{n}$, and all correlation coefficients $R^{2}$ are about 0.99 .

Hence the constitutive equation of HNFIII can be obtained:

$$
\begin{cases}\eta=a v(c) & \gamma \leq \gamma_{c} \\ \eta=k(c) \cdot \gamma^{n-1} & \gamma \geq \gamma_{c}\end{cases}
$$


Table 1. Coefficients and indexes of HNFIII constitutive equations of NFs.

\begin{tabular}{lllllllll}
\hline Concentration / wt\% & 0.2 & 0.15 & 0.1 & 0.075 & 0.05 & 0.02 & 0.01 & 0.0025 \\
\hline $\mathrm{AV}(\mathrm{c})$ & 1.898 & 1.533 & 1.427 & 1.207 & 1.137 & 1.003 & 1.246 & 1.026 \\
Coefficient $k$ & 0.11 & 0.089 & 0.088 & 0.087 & 0.086 & 0.083 & 0.082 & 0.077 \\
Index $n$ & 1.52 & 1.539 & 1.532 & 1.525 & 1.523 & 1.523 & 1.522 & 1.522 \\
$R^{2}$ & 0.991 & 0.994 & 0.994 & 0.995 & 0.995 & 0.996 & 0.994 & 0.994 \\
\hline
\end{tabular}

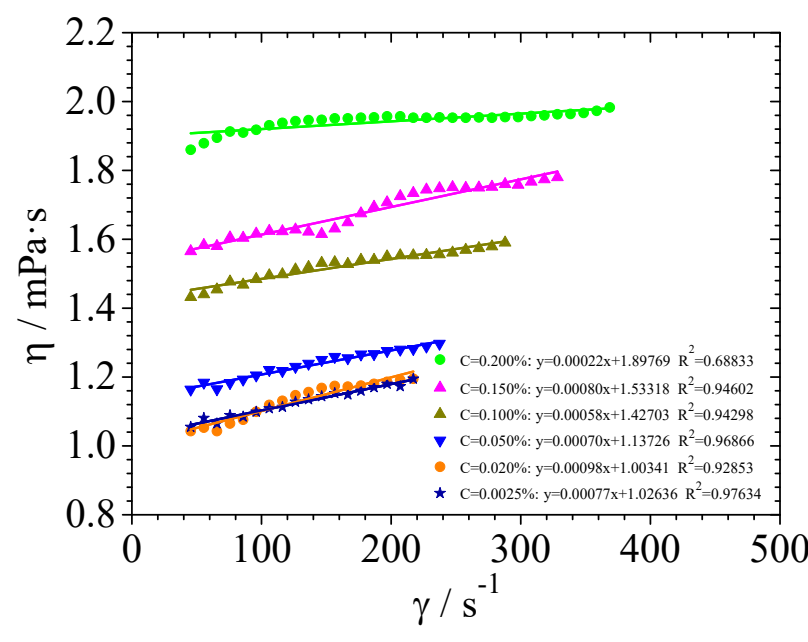

Fig. 3. Shear rate-AV curves of NFs HNFIII before the critical point.

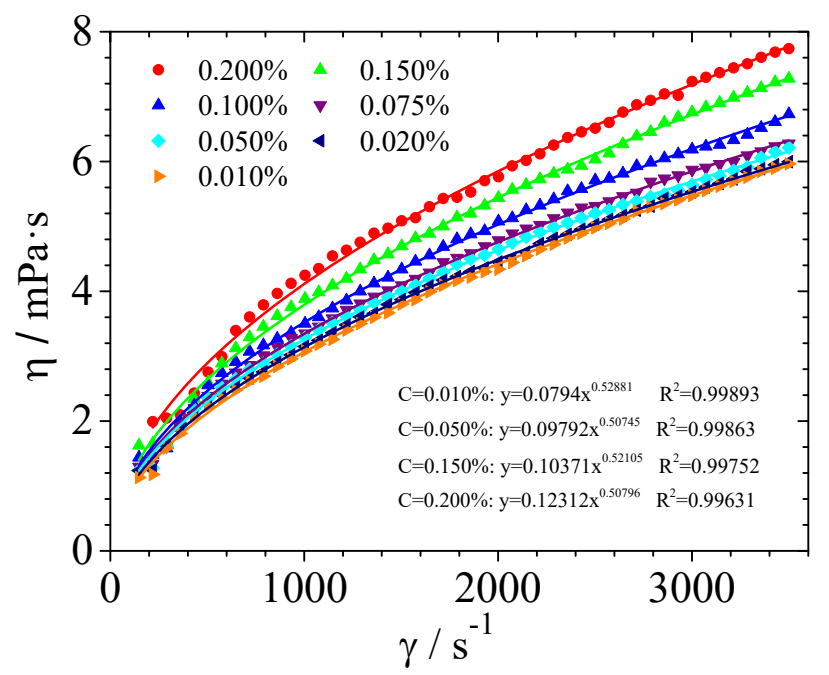

Fig. 4. Shear rate-AV curves of NFs after the critical point.

In Eq. (1), $a v(c), k(c)$ and $n$ are $\mathrm{AV}$, coefficient and index related to concentration, respectively. These values can be obtained by fitting the $\mathrm{AV}$-shear rate curves, as shown in Table 1.

The AV decreases with the concentration decrease at the Newtonian-like fluid stage. The coefficient is around 1.0 after the concentration is lower than $0.02 \mathrm{wt} \%$, indicating that the fluid in this case is close to water. It is noted that the value at $0.01 \mathrm{wt} \%$ is abnormal, which may relate to the test instability

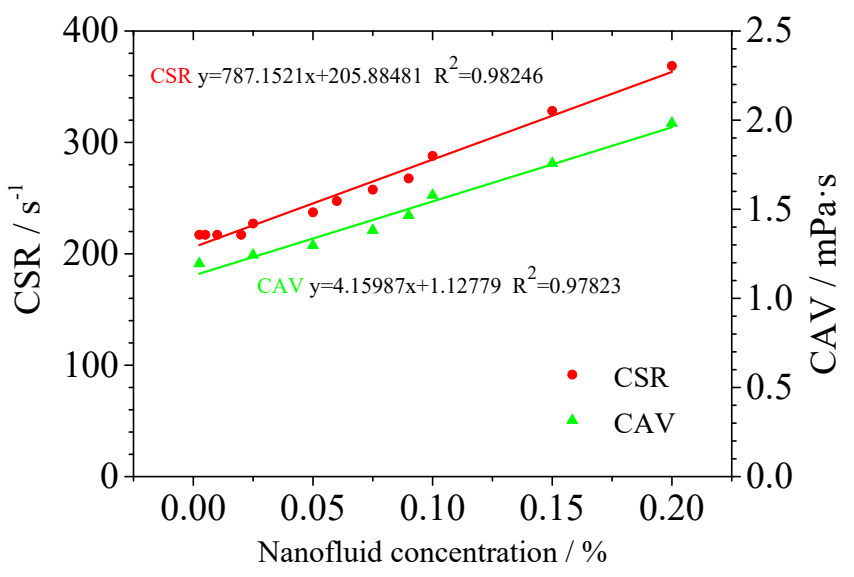

Fig. 5. The relationships between critical values and concentration of dragreducing NFs.

and is excluded. The AV has a good linear relationship with the concentration. The correlation equation is $a v(c)=$ $1.472 c+0.936$, and the correlation coefficient $R^{2}$ is 0.962 . The index $n$ is around 1.5 and larger than 1 in the nonNewtonian fluid stage, indicating that HNFIII is a power law fluid (expansion type). As the concentration decreases, the index varies irregularly and the coefficient decreases.

\subsection{Relationship between critical values and concentration of HNFIII}

Critical shear rate (CSR) and critical apparent viscosity $(\mathrm{CAV})$ are very important parameters at the boundary point of two-stage flow state of NFs, which are related to concentrations. The curves of the CSR $\gamma_{c}$ and CAV $\eta_{c}$ versus the concentration are plotted in Fig. 5. The two curves show a good linear correlation with the correlation coefficients of 0.97 and 0.933 , respectively. The relation equations are:

$$
\begin{gathered}
\gamma_{c}=787 c+205.9 \\
\eta_{c}=4.159 c+1.127
\end{gathered}
$$

In order to control the viscosity of NFs, the injection rate must be below the critical value. The critical shear rate provides a clear guidance on the injection rate of NFs. The critical shear rate or critical $\mathrm{AV}$ at different concentrations can be obtained by using Eq. (2) or Eq. (3). 


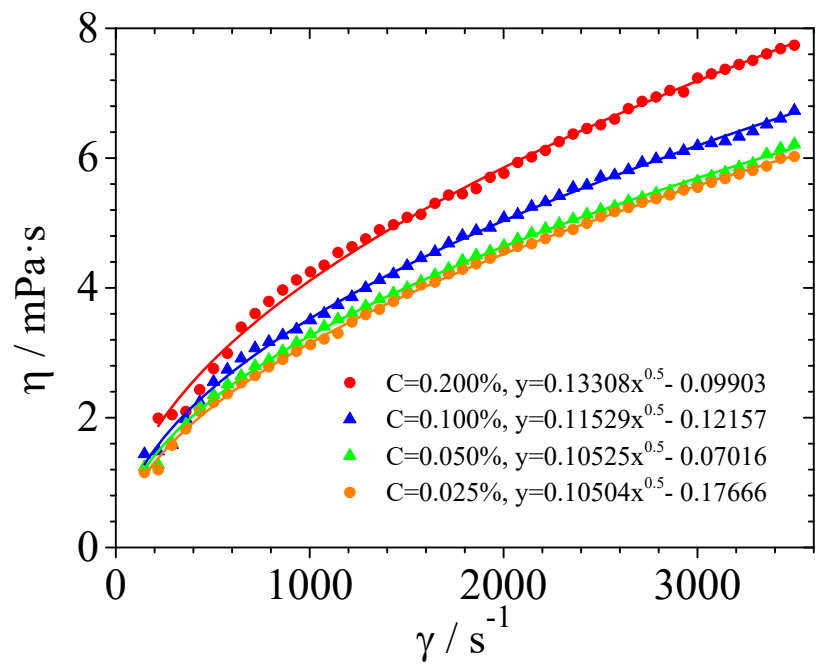

Fig. 6. The CICE of HNFIII in non-Newtonian segment.

\subsection{Construction of CICE in non-Newtonian stage}

As mentioned earlier, the index changes with the concentration, and the dimension of the coefficient also changes. The uncertainty of the coefficient dimension causes the confusion of physical meaning. Moreover, the equation cannot reflect the influence of the concentration. Theoretically for the same fluid, the dimension of each parameter should be determined. From Table 1, it is found that the indices $n$ are around 1.5 in the equations with different concentrations. Hence, assuming $n$ is 1.5, the data were fitted with coefficients to reflect the change in concentration, which is called fitting exponential method.

The "Fit function" provided by Mathematica was used to fit the data. A set of equations with an index of 0.5 were obtained. All the correlation coefficients are higher than 0.96 in Fig. 6. The general equation for these curves is given by:

$$
\mu=k(c) \gamma^{0.5}+b \quad\left(\gamma \geq \gamma_{c}\right)
$$

The parameter $b$ in Eq. (4) is very small or may be a test error, and this part of the value can be negligible when the shear rate exceeds the critical value. Or let $\mu=\mu-b$, so that the Eq. (4) can be written as:

$$
\mu=k(c) \gamma^{0.5}
$$

The coefficients of different concentrations of NFs in the CICE were fitted to the concentration curve to obtain the coefficient equation. The correlation coefficient was 0.964 , as shown in Fig. 7.

Finally, the relationship of viscosity, concentration and shear velocity is obtained as:

$$
\mu=(15.61 c+0.1) \gamma^{0.5} \quad\left(\gamma \geq \gamma_{c}\right)
$$

Eq. (6) corresponds to the CICE of HNFIII in nonNewtonian stage. The formula shows that the viscosity of the nanofluid is a constant exponential power function of shear rate with an exponent of 0.5 , and the coefficient is linearly

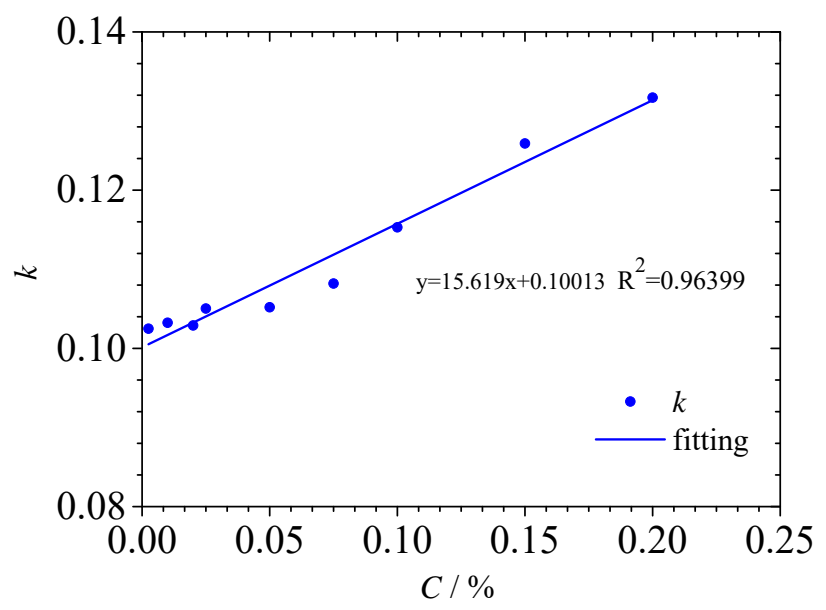

Fig. 7. The relationship between $k$ and $c$ in the CICE of HNFIII.

related to the concentration. Compared with the traditional non-Newtonian fluid constitutive equation, the advantages are as follows: first, the concentration is no longer an invisible factor, but a dominant parameter, which can directly reflect the characteristics of different concentrations; second, one equation replaces a set of equations, so the expression efficiency is improved; third, the index is determined, so the dimension of the coefficient is also determined, and there is no more confusion of physical meaning.

For convenience of application, the shear rate needs be transformed into flow rate. According to the parameters of rotor diameter and conversion coefficient $M$ of rotary rheometer, the relationship between average flow velocity and shear rate is as follows:

$$
v=\frac{d}{2 M} \cdot \gamma
$$

From Eqs. (6) and (7), we obtain:

$$
\mu=(510.16 c+3.27) v^{0.5} \quad\left(\gamma \geq \gamma_{c}\right)
$$

here, $v$ is flow velocity, $\mathrm{m} / \mathrm{s} ; d$ is the average diameter of the rotor and the stator, $0.042 \mathrm{~m} ; M$ is the conversion coefficient of the rotor, which is $22.43 \mathrm{~s}^{-1} /(\mathrm{rad} / \mathrm{s})$.

Eq. (8) is the CICE of NFs expressed using flow velocity.

The method is applied to two hydrophilic NFs, No. 2 and No. 3, and the CICE is obtained by taking the test data with $0.15 \%$ concentration, as shown in Fig. 8. The constitutive equations of the two NFs conform to constant exponential equation $\mu=k(c) \gamma^{0.5}$, and the correlation coefficients are 0.985 and 0.982 , respectively.

\subsection{Construction of dimensionless CICE}

In numerical simulations, dimensionless form is often presented to facilitate calculation and discussion. The CSR and the CAV are used as characteristic parameters for dimensionless treatment. The dimensionless variables are defined as: 


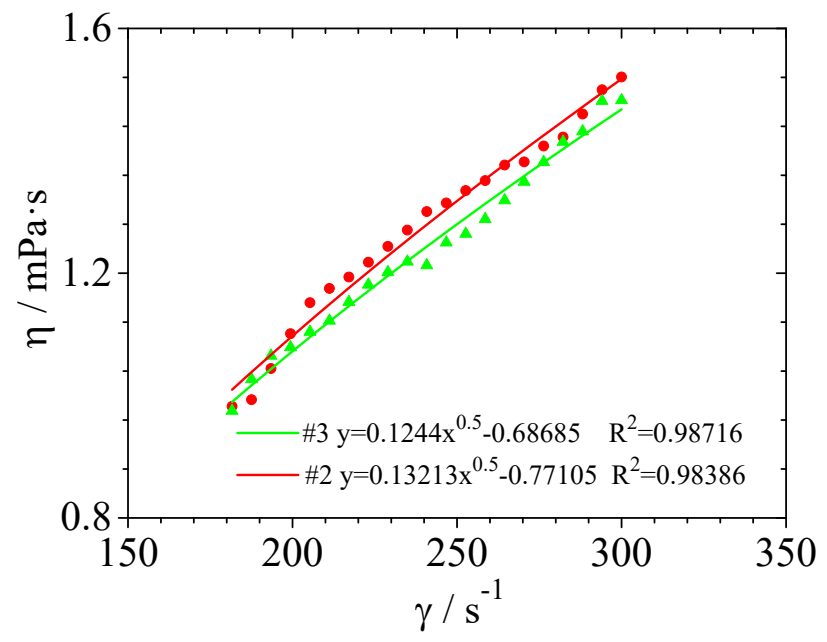

Fig. 8. \#2 and \#3 hydrophilic nano-fluid constant index constitutive equations $(0.15 \mathrm{wt} \%)$.

Table 2. Critical parameters of HNFIII.

\begin{tabular}{llll}
\hline$C / \%$ & $\gamma_{c} / \mathrm{s}^{-1}$ & $v_{c} / \mathrm{m} / \mathrm{s}$ & $\mu_{c} / \mathrm{mPa} \cdot \mathrm{s}$ \\
\hline 0.0025 & 217.1 & 0.203 & 1.195 \\
0.010 & 217.1 & 0.203 & 1.223 \\
0.025 & 237.3 & 0.213 & 1.243 \\
0.050 & 237.3 & 0.222 & 1.297 \\
0.075 & 257.6 & 0.241 & 1.382 \\
0.100 & 287.9 & 0.270 & 1.590 \\
0.150 & 338.4 & 0.317 & 1.828 \\
0.200 & 368.7 & 0.345 & 2.063 \\
\hline
\end{tabular}

$$
\gamma_{D}=\frac{\gamma}{\gamma_{c}}, v_{D}=\frac{v}{v_{c}}, \mu_{D}=\frac{\mu}{\mu_{c}}
$$

where $\gamma_{D}, v_{D}$ and $\mu_{D}$ are dimensionless shear rate, dimensionless velocity and dimensionless $\mathrm{AV}$, respectively.

First, the critical shear rate, critical velocity and critical viscosity at the inflection point are determined according to the curve. The results are shown in Table 2. Then, the critical parameters of each concentration are taken as the base point, and the dimensionless treatment is carried out according to Eq. (8). Finally, the processed data are plotted in Fig. 9.

It can be found that the dimensionless curves are very similar and almost coincide in Fig. 7, and the fitting curve can be obtained as:

$$
\mu_{D}=k_{D} v_{D}^{0.5}
$$

According to the fitting results, it is found that $k_{D}$ is 1.125 1.206, which is almost the same. That is, the dimensionless constitutive equation is almost independent of the concentration, which unifies the equations of different concentrations. The intrinsic reason is still to be investigated.

\section{Conclusions}

The rheological properties of several NFs were measured to obtain the non-Newtonian types and critical parameters. The

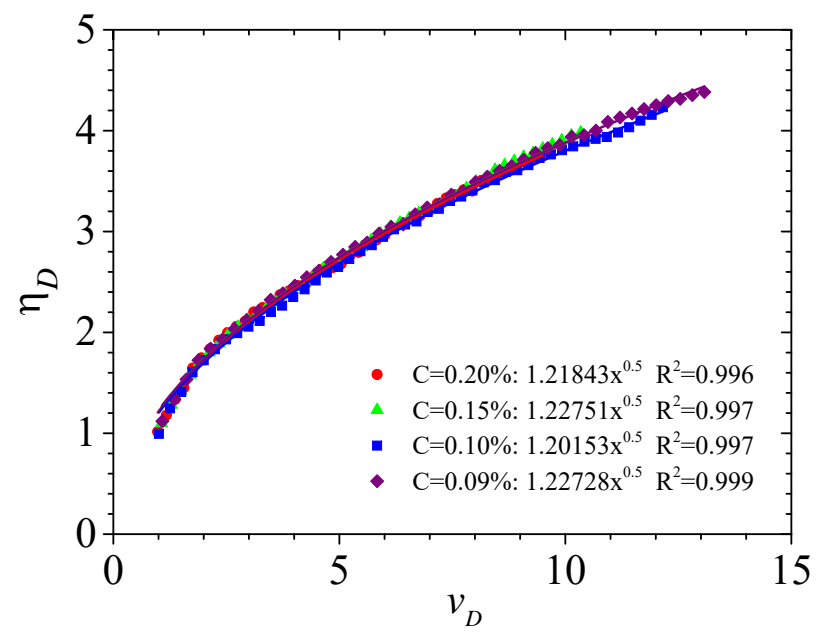

Fig. 9. Relationship between dimensionless velocity and dimensionless viscosity.

shear thickening mechanism is investigated by two-way experiment of increasing and decreasing shear rate. A comprehensive explanation of order or disorder theory and particle cluster theory is given. A CICE is established using the fixed index method. The following conclusions can be drawn from this study:

All the tested NFs are complex non-Newtonian fluids, which are combination of newtonian-like and power-law types. The NFs have shear thickening properties and critical shear rates. The AV of NFs show a slow linear increase with the shear rate below the critical value, while the shear thickening characteristic of power-law fluid appears after the critical shear rate is exceeded. The critical shear rate shows linear increasing relationship with the concentration.

The shear thickening mechanism of NFs is that the particle aggregation is caused by the separation of particles and base liquid due to high velocity. Under high shearing, the structure is subject to a transient physical change. The "particle cluster" of NFs with uniform order and particle transient aggregation are two different stages of NFs rheological behaviour.

A CICE of non-Newtonian fluid with an exponent of 0.5 is constructed and its coefficient $k(c)$ has a linear relation with the concentration. The CICE is simple and can replace a set of conventional constitutive equations with different concentrations. The coefficients dimension is invariant with different concentrations, so the physical meaning is clarified. It also provides a new method for constructing constitutive equations of non-Newtonian fluids.

The dimensionless CICE can unify the equations of different concentrations. It will guide the applications of the dimensionless CICE to drag-reducing NFs flow modeling in porous media.

\section{Acknowledgement}

This work is supported partly by the National Natural Science Foundation of China (51274136, 51704190), and Shanghai Leading Academic Discipline Project (S30106) and the Shanghai Municipal Education Commission Discipline 
Construction Program.

\section{Conflict of interest}

The authors declare no competing interest.

Open Access This article, published at Yandy Scientific Press on behalf of the Division of Porous Flow, Hubei Province Society of Rock Mechanics and Engineering, is distributed under the terms and conditions of the Creative Commons Attribution (CC BY-NC-ND) license, which permits unrestricted use, distribution, and reproduction in any medium, provided the original work is properly cited.

\section{References}

Aladag, B., Halelfadl, S., Doner, N., et al. Experimental investigations of the viscosity of nanofluids at low temperatures. Appl. Energy 2012, 97: 876-880.

Alnarabiji, M.S., Husein, M.M. Application of bare nanoparticle-based nanofluids in enhanced oil recovery. Fuel 2020, 267: 117262.

Alphonse, P., Bleta, R., Soules, R. Effect of PEG on rheology on stability of nanocrystalline titania hydrosols. J. Colloid Interface Sci. 2009, 337(1): 81-87.

Assef, Y., Arab, D., Pourafshary, P. Application of nanofluid to control fines migration to improve the performance of low salinity water flooding and alkaline flooding. J. Pet. Sci. Eng. 2014, 124: 331-340.

Baghbanzadeh, M., Rashidi, A., Soleimanisalim, A.H., et al. Investigating the rheological properties of nanofluids of water/hybrid nanostructure of spherical silica/MWCNT. Thermochim. Acta 2014, 578: 53-58.

Bossis, G., Brady, J.F. The rheology of Brownian suspensions. J. Chem. Phys. 1989, 91(3): 1866-1874.

Cao, S., Zhang, D., Shi, L., et al. The micro-dispersion research of nano-augmented injection microemulsion. Journal of Function Materials 2010, 41(12): 2210-2212. (in Chinese)

Catherall, A.A., Melrose, J.R., Ball, R.C. Shear thickening and order-disorder effects in concentrated colloids at high shear rates. J. Rheol. 2000, 44(1): 1-25.

Chan, C.F., Mong, F. Some constitutive equations for nonNewtonian fluids. Chinese Journal of Theoretical and Applied Mechanics 1983, 19(1): 16-26. (in Chinese)

Cheng, Y., Li X., Li Q., et al. Preparation and performance of water-based nanometer polysilicon as augmented injection for oil field. Chemical Research 2006, 17(4): 56-59. (in Chinese)

Chen, H., Ding, Y. Heat transfer and rheological behaviour of nanofluids-A review. in Advances in Transport Phenomena, edited by L.Q. Wang, Springer, Berlin, Heidelberg, pp. 135-177, 2009.

Chen, H., Di, Q., Ye, F., et al. Numerical simulation of drag reduction effects by hydrophobic nanoparticles adsorption method in water flooding processes. J. Nat. Gas Sci. Eng. 2016, 35: 1261-1269.

Das, S.K., Putra, N., Thiesen, P., et al. Temperature dependence of thermal conductivity enhancement for nanofluids. J. Heat Transf. 2003, 125(4): 567-574.

Ettefaghi, E., Rashidi, A., Ahmadi, H., et al. Thermal and rheological properties of oil-based nanofluids from different carbon nanostructures. Int. Commun. Heat Mass Transf. 2013, 48: 178-182.

Gu, C., Di, Q., Fang, H. Slip velocity model of porous walls absorbed by hydrophobic nanoparticles $\mathrm{SiO}_{2}$. J. Hydrodyn. 2007, 19(3): 365-371.

Gu, C., Di, Q., Shi, L., et al. Drag reduction technology with nanomaterial ShUNP2-10 in oilfield. Adv. Mater. Res. 2013, 807-809: 2492-2497.

Heine, D.R., Petersen M.K., Grest G.S. Effect of particle shape and charge on bulk rheology of nanoparticle suspensions. J. Chem. Phys. 2010, 132(18): 184509.

He, Y., Jin, Y., Chen, H.S., et al. Heat transfer and flow behaviour of aqueous suspensions of $\mathrm{TiO}_{2}$ nanoparticles (nanofluids) flowing upward through a vertical pipe. Int. J. Heat Mass Transf. 2007, 50(11-12): 2272-2281.

Hong, R.Y., Ren, Z.Q., Han, Y.P., et al. Rheological properties of water-based $\mathrm{Fe}_{3} \mathrm{O}_{4}$ ferrofluids. Chem. Eng. Sci. 2007, 62(21): 5912-5924.

$\mathrm{Hu}, \mathrm{X} ., \mathrm{Li}, \mathrm{S}$., Wu, H. The application of poly-silicon manoaugmented injection technology in high-salt sensitivity reservoirs in Jiangling depression. Journal of Oil and Gas Technology 2012, 34(7): 129-131. (in Chinese)

Jóźwiak, B., Boncel, S. Rheology of ionanofluids-A review. J. Mol. Liq. 2020, 302: 112568.

Ju, B.S., Dai S.G., Luan Z.A., et al. A study of wettability and permeability change caused by adsorption of nanometer structured polysilicon on the surface of porous media. Paper SPE 77938 Presented at SPE Asia Pacific Oil and Gas Conference and Exhibition, Melbourne, Australia, 8-10 October, 2002.

Lu, K. Rheological behaviour of carbon nanotube-alumina nanoparticle dispersion systems. Powder Technol. 2007, 177(3): 154-161.

Lu, X., Lv, G., Luan, Z., et al. Application of polysilicon in low permeability oil field. Petroleum Exploration and Development 2003, 30(6): 110-122. (in Chinese)

Maranzano, B.J., Wagner, N.J. The effect of particle size on reversible shear thickening of concentrated colloidal dispersions. J. Chem. Phys. 2001, 114(23): 10514-10527.

Maranzano, B.J., Wagner, N.J. Flow-small angle neutron scattering measurements of colloidal dispersion microstructure evolution through the shear thickening transition. J. Chem. Phys. 2002, 117(22): 10291-10302.

Moattar, M.T.Z., Cegincara, R.M. Stability, rheological, magnetorheological and volumetric characterizations of polymer based magnetic nanofluids. Colloid Polym. Sci. 2013, 291(8): 1977-1987.

Moghadasi, R., Rostami, A., Hemmati-Sarapardeh, A. Application of nanoflfluids for treating fines migration during hydraulic fracturing: Experimental study and mechanistic understanding. Adv. Geo-Energy Res. 2019, 3(2): 198206.

Moghaddam, M.B., Goharshadi, E.K., Entezari,, M.H., et al. Preparation, characterization and rheological properties of grapheme-glycerol nanofluids. Chem. Eng. J. 2013, 231: $365-372$. 
Mohamadian, N., Ghorbani, H., Wood, D.A., et al. Rheological and filtration characteristics of drilling fluids enhanced by nanoparticles with selected additives: An experimental study. Adv. Geo-Energy Res. 2018, 2(3): 228-236.

Muhammad, Z.H., Daisuke, H., Akira, Y., et al. Dispersion and rheology of nanofluids with various concentrations of organic modified nanoparticles: Modifier and solvent effects. Colloid Surf. A 2019, 583: 123876.

Navarrete, N., Hernandez, L., Vela, A., et al. Influence of the production method on the thermophysical properties of high temperature molten salt-based nanofluids, J. Mol. Liq. 2020, 302: 112570.

Pang, D., Gu, C., Di, Q., et al. Research of rheology of friction-reducing nano silicon dioxide fluid. Journal Xian Shiyou University (Natural Science Edition) 2016, 31(5): 78-83. (in Chinese)

Petel, O.E., Ouellet, S., Loiseau, J., et al. The effect of particle strength on the ballistic resistance of shear thickening fluids. Appl. Phys. Lett. 2013, 102(6): 064103.

Sharma, A.K., Tiwari, A.K., Dixit, A.R. Rheological behaviour of nanofluids: A review. Renew. Sust. Energ. Rev. 2016, 53: 779-791.

Sha, X. Preparation and properties research of shear thicking liquid. Wuxi, Jiangnan University, 2013. (in Chinese)

Susan-Resiga, D., Socoliuc, V., Boros, T., et al. The influence of particle clustering on the rheological properties of highly concentrated magnetic nanofluids. J. Colloid Interface Sci. 2012, 373(1): 110-115.
Wang, J., Wang, Z., Lei, S., et al. Research on the influence of different pretreatment technology on augmented injection effect of nanometer polysilicon material. Petrochemical Industry Application 2012, 31(2): 76-79. (in Chinese)

Wang, J., Zhu, J., Zhang, X., et al. Heat transfer and pressure drop of nanofluids containing carbon nanotubes in laminar flows. Exp. Therm. Fluid Sci. 2013, 44: 716-721.

Wang, L., Chen, H., Witharana, S. Rheology of nanofluids: A review. Recent Pat. Nanotechnology 2013, 7(3): 232-246.

Wang, X., Xu, X., Choi, S. Thermal conductivity of nanoparticle-fluid mixture. J. Thermophys. Heat Transf. 1999, 13(4): 474-480.

Yang, Y., Grulke, E.A., Zhang, Z.G., et al. Thermal and rheological properties of carbon nanotube in oil dispersions. J. Appl. Phys. 2006, 99(11): 114307.

Yuan, S., Wang Q. New progress and prospect of oilfields development technologies in China. Pet. Explor. Dev. 2018, 45(4): 698-711.

Yue, Y. Development of a new water-based nano-polysilicon injection enhance. Chengdu, Southwest Petroleum University, 2014. (in Chinese)

Yu, Q., Zheng N., Song, J., et al. Application of water-based nano-polysilicon emulsion. Oilfield Chemistry 2012, 29(2): 181-185. (in Chinese)

Zhao, M., Lv, W., Li, Y., et al. Study on the synergy between silica nanoparticles and surfactants for enhanced oil recovery during spontaneous imbibition. J. Mol. Liq. 2018, 261: 373-378. 